\title{
DER NEUE VERFASSUNGSENTWURF DER VOLKSREPUBLIK CHINA
}

\author{
Von Oskar Weggel und Y. H. Nieh
}

Seit ihrer Gründung im Jahre 1949 ist die Volksrepublik China ein sich ständig prüfendes, neu definierendes und wieder verwerfendes Gebilde. Trotz dieser permanenten inneren Bewegung aber war der Verfassungstext von 1954 unberührt geblieben - mit der Folge, daß viele Bestimmungen nur noch Fassade waren. Es bedurfte einer Erschütterung wie der Kulturrevolution, ehe auch dieses Zeugnis einer vergangenen Epoche zum Einsturz kam. 21 Jahre nach Ausrufung der Volksrepublik und eineinhalb Jahre nach Erlaß einer neuen Parteisatzung ist nun endlich die Novellierung der Verfassungsurkunde entscheidungsreif geworden. Ein Entwurf der neuen Konstitution wurde am 5. November 1970 von der nationalchinesischen "Zentralzeitung" (Chung-yang jih-pao) veröffentlicht. Dieses Dokument verdient Aufmerksamkeit, da es eine juristische Bestandsaufnahme und Legalisierung der durch die Kulturrevolution und den Sieg Mao Tse-tungs erzielten Ergebnisse liefert.

Die neue Verfassung ist wie die alte in vier Abschnitte unterteilt, nämlich I. Allgemeines Programm, II. Staatsorgane, III. Grundrechte und Grundpflichten, IV.Staatssymbole.

Der Entwurf hält auf den ersten Blick einige verfassungsrechtliche Kuriositäten: Mao Tse-tung wird schon gleich in Art. 2 als "großer Führer" und „Oberbefehlshaber" hervorgehoben. Lin Piao gilt als sein "enger Waffenfreund und Nachfolger" sowie als „stellvertretender Oberbefehlshaber". Gleichzeitig fiel das Amt des Staatspräsidenten, das der verfemte Liu Shao-ch'i jahrelang bekleidet hatte, dem Rotstift zum Opfer. (Art. 2 spricht zwar vom „Vorsitzenden“ Mao, präzisiert aber nicht, ob damit der „Vorsitzende“ des Zentralkomitees der Kommunistischen Partei oder der „Vorsitzende“ des Staates gemeint ist.) Mit dem Amt des Staatspräsidenten entfielen auch die beiden wichtigsten ihm früher zugeordneten Organe: die "Oberste Staatskonferenz" und der "Nationale Verteidigungsrat". Offensichtlich soll die Stellung des ersten Mannes im Staate so sehr auf die Person Maos und seines „Nachfolgers" Lin Piao zugeschnitten werden, daß auf spätere Anwärter kein Gedanke verwandt zu werden brauchte. Angesichts einer solchen Konstellation bedurfte es auch keiner Bestimmungen über Bestellung (im Gegensatz zu bisher ist keine Wahl mehr erforderlich!), Absetzung, Amtsdauer und vor allem auch nicht über die Zuständigkeit des obersten Repräsentanten.

Trug die alte Verfassung von 1954 die Handschrift Liu Shao-ch'is, so ist der nun vorliegende Entwurf von 1970 ganz von Mao Tse-tungs Vorstellungen geprägt:

Den ungleich „politischeren“ Charakter der neuen Konstitution deutet schon das äußere Rahmenwerk an: Hatte das alte Grundgesetz noch 106 Artikel (mit rund 14000 Wortzeichen) umfaßt, so genügen nunmehr 30 Artikel (mit ungefähr 3400 Zeichen). Hatten die vor allem politische Grundsätze enthaltenden „Allgemeinen Bestimmungen" früher nur ein Fünftel des Verfassungstextes eingenommen, so ist es nunmehr ziemlich genau die Hälfte. Nicht nur in der äußeren Aufmachung aber, sondern vor allem in ihrem inneren Gehalt wurde die Verfassung "maoisiert". „Politische Arbeit“ - und nicht so sehr fachliches Können - gehört nun zur 
„Lebenslinie“ aller Arbeit. Die „Mao-Tse-tung-Ideen“ sind „lebendig zu lernen und anzuwenden“. Es gilt, die „proletarische Politik in den Vordergrund zu stellen“, den „Bürokratismus zu bekämpfen“ und „sich mit den Massen aufs engste zu verbinden “. „Großes Singen und Blühen" (gemeint ist offene Meinungsäußerung), „große Wandzeitungen" und "große Diskussionen" sollen der Selbsterziehung der Massen dienen. Schon aus diesen wenigen Bestimmungen wird ersichtlich, daß es sich bei dem neuen knappen Entwurf um eine Verfassung der Generalklauseln handelt, deren näherer Gehalt erst durch die schöpferische Spontaneität der Volksmassen, d. h. also im Wege der Praxis zu ermitteln ist.

Überall kommen hier die Grundsätze der Kulturrevolution wieder zu Wort. Besonders deutlich wird dies, wenn die Verfassung Verwaltungsvereinfachung und die „Dreierallianz“ zwischen Funktionen, Soldaten und Massenvertretern sowie zwischen „Alt, Mittel und Jung“ befürwortet. Eine drei Jahre alte Realität wird damit endlich legalisiert. Gleichzeitig ist die maoistische Idee, daß jeder chinesische Staatsbürger zur selben Zeit Soldat, Bauer, Arbeiter und Verwalter sein sollte, juristisch fixiert worden. Auch die Definition der Volksrepublik als „sozialistischer Staat" (und nicht mehr - wie 1954 - als „Volksdemokratie“), die Einschränkung des Eigentums an Produktionsmitteln auf zwei Formen, nämlich Volkseigentum und Kollektiveigentum, der Wegfall aller früheren Bestimmungen, die der sogenannten Bourgeoisie Existenz, Vermögen und Erbrecht garantierten, die Betonung des Klassenkampfcharakters des Staates und des permanenten Charakters der Revolution sowie schließlich die Eliminierung aller Bestimmungen, die einen Parteienpluralismus zulassen, sind deutliche Anzeichen dafür, daß sich die Verhältnisse grundsätzlich geändert haben. War die Konstitution von 1954 noch stark an der Sow jetverfassung Stalins von 1936 orientiert, so zeigt sie sich nun in durch und durch „sinisierter" Form. Die Abkehr von Moskau wird auch darin deutlich, daß der Konflikt mit der Sowjetunion in den Katalog der Staatsaufgaben übernommen wurde.

Wie ernst es der Pekinger Führung gerade mit der Revolutionierung der Staatsapparatur ist, geht vor allem aus der radikalen Kürzung des Abschnittes II hervor, in dem die "Staatsorgane“ abgehandelt werden. Wo früher 64 Artikel notwendig waren, genügen heute 7. Anstelle der 6 alten Organe (Nationaler Volkskongreß, Staatspräsident, Staatsrat, örtliche Volksversammlungen und Volksausschüsse, Selbstverwaltungskörperschaften, Volksgerichte sowie Volksstaatsanwaltschaft) existieren - nach Abschaffung des Präsidentenamtes - nur noch 5 und auch diese nur noch in stark veränderter Form.

- Der Nationale Volkskongreß hat seine Stellung als „oberstes Organ der Staatsgewalt" sowie seine wichtigsten Rechte (Verfassungsänderung, Gesetzeserlaß, Haushalt, Bestellung des Staatsrats usw.) im wesentlichen behalten. Dasselbe läßt sich von seinem Ständigen Ausschuß, dem bisher faktisch machtvollsten Staatsorgan, behaupten; zusätzlich wird ihm nun noch das Recht auf Empfang auswärtiger Gesandter übertragen. Diese Befugnis war bisher dem Staatspräsidenten vorbehalten. Sollte also Mao Tse-tung in Zukunft noch einen Gesandten zusätzlich empfangen, so wäre dies ein Akt ganz besonderer Ehrung.

- Im wesentlichen unverändert blieb auch Chou En-lais Staatsrat, der zwar ziemlich farblos definiert wird, nach wie vor aber die "Zentrale Volksregierung“ verkörpert. Neu ist hier nur die Bestimmung, daß der Staatsrat den Nationalen Wirtschaftsplan sowie den Haushaltsplan „formuliert und ausführt“ (Art. 20). Früher durfte er nur „Maßnahmen zur Realisierung der Wirtschaftspläne und 
zur Durchführung des Staatshaushalts treffen“ (Art. 49, 7 der Verfassung von 1954). Damals entschied nämlich der Nationale Volkskongreß über die Volkswirtschaftspläne und prüfte den Staatshaushalt. Aus der Novellierung wird allerdings nicht recht klar, wieweit sich die Planungs- und Haushaltsbefugnisse des Staatsrats wirklich geändert haben.

- Welchen Einfluß die Kulturrevolution auf die Organstruktur ausgeübt hat, zeigt sich freilich erst im regionalen Bereich, dessen Partei- und Volksausschüsse in den Wirren der Jahre nach 1966 weitgehend zerstört worden waren. Hier sollen in Zukunft die Revolutionskomitees als ständige regionale Regierungen fungieren und als solche den örtlichen Volkskongressen verantwortlich sein. Wirkliche Befehlsgewalt dürften freilich auch in Zukunft die lokalen Parteiausschüsse ausüben. Was die Revolutionskomitees von den bisherigen lokalen Volksausschüssen unterscheidet, ist vor allem ihre Mission als „revolutionierte" Organe, in denen der Bürokratismus keinen Halt mehr findet. Regelmäßige Teilnahme der Funktionäre an der Produktionsarbeit und „enge Verbindung mit den Massen“ sollen dafür garantieren, daß die Revolutionskomitees ihren Rätecharakter bewahren.

- Das Selbstverwaltungsrecht der Minoritäten wird zwar garantiert und soll durch Volkskongresse und Revolutionskomitees ausgeübt werden. Die Verfassung verurteilt jedoch gleichzeitig jede Form eines "lokalen Nationalismus“, erklärt auch Minderheitengebiete als "unabtrennbar" (Art. 4) und bestimmt Mao Tse-tungs Ideen zur eigentlichen Richtlinie. Damit ist sichergestellt, daß sich eventuelle Separationsbewegungen als Auswirkung von Klassenkämpfen und nicht etwa von völkischen Spannungen - behandeln lassen.

- Stiefmütterlich sind Justiz und Staatsanwaltschaft geregelt. In diesem Zusammenhang wird erneut deutlich, wie sehr sich diese Funktionsbereiche, die ja neben der Polizei ganz unter den Einfluß Liu Shao-ch'is geraten waren, die Gunst Maos verscherzt haben. Von Unabhängigkeit der Gerichte ist heute im Gegensatz zur alten Verfassung - nirgends mehr die Rede. „Wichtige kriminelle und konterrevolutionäre Fälle" sollen im Wege der Massendiskussion entschieden werden, wie überhaupt die "Massenlinie“ das gesamte Organisationswesen wie ein roter Faden durchzieht.

Kein Zufall ist es, daß ausgerechnet die Armee mit der Aufgabe betraut ist, die Nation nicht nur vor dem äußeren Feinde zu beschützen, sondern gleichzeitig auch $\mathrm{zu}$ helfen, den „sozialistischen Aufbau“ zu verteidigen. Ihre in der Kulturrevolution errungene einzigartige Machtstellung wird in dieser großzügigen Formulierung erneut deutlich. Daß die Armee auch "Arbeitstruppe“ ist (d. h. auch für politischpropagandistische Tätigkeiten einzustehen hat), bestätigt nur einmal mehr ihre Stellung als "Schule der Nation“.

Bemerkenswert ist schließlich, daß die Kommunistische Partei, die 1954 nur in der Präambel erwähnt worden war, im Entwurf gleich fünfmal auftaucht (Art. 1, 15, 16, 17, 26). Wie vor allem aus Art. 1 und 16 hervorgeht, steht die Partei über dem Staat. Auch bricht Parteirecht (die Parteisatzung von 1969) entgegenstehendes Verfassungsrecht. Hoch über der Partei wiederum aber steht Mao Tse-tung und sein Denken (Art. 2, 11, 26).

Der Text ist ein rein innenpolitisches Dokument. Abgesehen von Art. 18, der die Berechtigung zur Ratifizierung völkerrechtlicher Verträge regelt, taucht das Ausland nur in der Präambel auf, wo die Feinde („Imperialismus, Sozio-Imperialismus 
und ihre Lakaien“) genannt sind (Art. 15). Mit dem Ausdruck „Sozio-Imperialismus" ist auf die Sowjetunion angespielt.

Abschnitt III enthält die "Grundrechte und Grundpflichten der Staatsbürger“. Wie in der alten Verfassung sind hier die üblichen formalen Rechte (Wahlrecht, Freiheit der Meinung, der Vereinigung, der Demonstration, der Religion usw.) aufgezählt, deren relativer Wert vor allem während der Kulturrevolution deutlich geworden ist. Das „Recht zum Streiken“ (Art. 28) ist neu, ohne daß ihm freilich in der Praxis Bedeutung zukäme.

Niemand wird es überraschen, daß als wichtigste Grundpflicht - und zugleich als Grundrecht! - „die Unterstützung des Vorsitzenden Mao und seines engen Waffengefährten Lin Piao" genannt ist. Jeder einzelne hat daneben die Aufgabe, für die Kommunistische Partei, die Diktatur des Proletariats, das sozialistische System und für die Gesetzesordnung der Volksrepublik einzutreten.

Wenig ist nach alledem von der alten Verfassung geblieben. Nur die Festlegung der Nationalflagge, des Staatswappens und der Hauptstadt ist unverändert übernommen worden. Im übrigen aber erinnert der Entwurf an einen Abschnitt aus der Mao-Bibel: Die Kulturrevolution hat eine neue Magna Charta erhalten.

\section{Entwurf einer Verfassung der Volksrepublik China ${ }^{1}$ (nach Chung-yang jih-pao vom 5. November 1970)}

\section{Abschnitt I: Allgemeine Grundsätze}

\section{Artikel 1}

Die Volksrepublik China ist ein sozialistischer Staat unter der Diktatur des Proletariats, der von der Arbeiterklasse (durch die KP) geführt wird und auf dem Bündnis zwischen Bauern und Arbeitern beruht.

\section{Artikel 2}

Der Vorsitzende Mao Tse-tung ist der große Führer aller Nationalitäten im ganzen Staat, er ist Oberhaupt unseres Staates der Diktatur des Proletariats, er ist Oberbefehlshaber der gesamten Nation und aller Streitkräfte. Stellvertretender Vorsitzender Lin ist der enge Waffenfreund und Nachfolger des Vorsitzenden Mao, er ist stellvertretender Oberbefehlshaber des ganzen Staates und aller Streitkräfte. Mao Tse-tungs Denken ist die Richtlinie für alle Arbeit des Volkes der ganzen Nation.

\section{Artikel 3}

Alle Macht in der Volksrepublik China gehört dem Volk. Die Organe, durch die das Volk seine Macht ausübt, sind die Volkskongresse aller Ebenen, auf denen hauptsächlich Arbeiter, Bauern und Soldaten als Abgeordnete vertreten sind. Die Volkskongresse aller Ebenen und die anderen Staatsorgane verwirklichen das Prinzip des demokratischen Zentralismus. Abgeordnete für die Volkskongresse aller Ebenen werden durch demokratische Konsultation gewählt. Die ursprünglichen Wahlgremien und die Wähler haben das Recht, die von ihnen gewählten Deputierten zu kontrollieren und, in Übereinstimmung mit den Gesetzesbestimmungen, sie jederzeit abzuberufen oder zu ersetzen.

1 Ubersetzung der Verfasser. 


\section{Artikel 4}

Die Volksrepublik China ist ein aus vielen Nationalitäten bestehender Einheitsstaat. Die Gebiete, in denen sich die nationalen Minderheiten selbst verwalten, sind unabtrennbare Teile der Volksrepublik China. Alle Nationalitäten sind gleichberechtigt; nationaler Groß-Chauvinismus und lokaler Nationalismus sind unzulässig; allen Nationalitäten steht es frei, ihre eigene Sprache und Schrift anzuwenden.

\section{Artikel 5}

Gegenwärtig zerfällt das Eigentum an Produktionsmitteln in der Volksrepublik China hauptsächlich in zwei Kategorien: sozialistisches Eigentum durch das ganze Volk und sozialistisches Kollektiveigentum durch die Massen des arbeitenden Volkes. Der Staat erlaubt allen nicht in der Landwirtschaft tätigen Arbeitern, unter der vereinigten Anleitung von Straßenkomitees in Städten und Märkten sowie von Produktionsmannschaften in Volkskommunen einer individuellen Tätigkeit nachzugehen, vorausgesetzt, daß sie sich im Rahmen der Gesetze hält und daß andere nicht ausgebeutet werden. Gleichzeitig sollen sie dazu angeleitet werden, nach und nach den Weg der sozialistischen Kollektivierung zu beschreiten.

\section{Artikel 6}

Der Staatssektor der Wirtschaft ist die führende Kraft in der Volkswirtschaft. Alle Bodenschätze, Gewässer und staatliche Waldungen, brachliegende Ländereien und andere natürliche Reichtümer sind Eigentum des ganzen Volkes. Der Staat ist berechtigt, nach Maßgabe der gesetzlich vorgesehenen Bestimmungen städtische und dörfliche Grundstücke sowie anderes Produktionskapital zwangsweise in Gebrauch $\mathrm{zu}$ nehmen oder zu verstaatlichen.

\section{Artikel 7}

Die bäuerlichen Volkskommunen sind Organisationen, in denen Regierung und Gesellschaft vereinigt sind. Gegenwärtig ist die Wirtschaft des kollektiven Eigentums in den ländlichen Volkskommunen im allgemeinen in drei Eigentumsstufen verwirklicht, nämlich: Eigentum der Volkskommune, wobei die Produktionsmannschaften als Grundverrechnungseinheiten fungieren, ferner Produktionsbrigaden und Produktionsmannschaften. Unter der Voraussetzung, daß die Kollektivwirtschaft der Volkskommune in ihrer Entwicklung gesichert bleibt und daß sie die absolute Priorität einnimmt, ist es den Volkskommunemitgliedern gestattet, kleine Stücke Land für sich selbst zu bebauen.

\section{Artikel 8}

Sozialistisches öffentliches Eigentum ist unverletzbar. Der Staat garantiert die Konsolidierung und Entwicklung der sozialistischen Wirtschaft. Er verbietet es jedermann, mit irgendwelchen Mitteln die Interessen der sozialistischen Wirtschaft und des öffentlichen Eigentums zu schädigen.

\section{Artikel 9}

Der Staat geht von folgenden sozialistischen Prinzipien aus: „Wer nicht arbeitet, soll auch nicht essen“, „jeder nach seinen Fähigkeiten“ und „Verteilung nach Maßgabe der Arbeitsleistung". Der Staat beschützt das Arbeitseinkommen, die Ersparnisse, die Wohnung und das Recht auf andere Mittel der Lebenshaltung der Bürger. 


\section{Artikel 10}

Politische Arbeit ist die Lebenslinie aller Arbeit. Der Staat sorgt dafür, daß die Revolution angepackt und die Produktion vorangetrieben wird, daß gearbeitet wird, daß die Vorbereitung für den Kriegsfall betrieben wird, daß die sozialistische Wirtschaft vorankommt, daß sich die Entwicklung planmäßig und proportional vollzieht, damit auf der Grundlage des ununterbrochenen Anstiegs der gesellschaftlichen Produktivität das materielle und kulturelle Leben des Volkes nach und nach verbessert und die Unabhängigkeit sowie die Sicherheit des Staates gefestigt wird.

\section{Artikel 11}

Alle Mitarbeiter in den Staatsorganen und anderen Organen haben die Mao-Tsetung-Ideen lebendig zu studieren und lebendig anzuwenden. Sie haben besonderen Wert auf proletarische Politik zu legen, dem Bürokratismus Widerstand zu leisten, sich mit Arbeitern, Bauern und den übrigen arbeitenden Massen zusammenzuschließen und dem Volk aus ganzem Herzen zu dienen. Das Arbeitspersonal aller Organe hat ständig an kollektiver Arbeit teilzunehmen. Alle Staatsorgane haben dem Grundsatz der Verwaltungsvereinfachung zu genügen; ihre Führungsorgane haben die revolutionäre Dreier-Allianz zwischen Armeevertretern, Funktionären und Massen sowie zwischen Alt, Mittel und Jung herbeizuführen.

\section{Artikel 12}

Das Proletariat hat im Úberbau, $z u$ dem auch die verschiedenen kulturellen Gebiete gehören, über die Bourgeoisie eine umfassende Diktatur auszuüben. Kultur, Erziehung, Literatur, Kunst und wissenschaftliche Forschung haben ausnahmslos im Dienste der proletarischen Politik sowie im Dienste der Arbeiter, Bauern und Soldaten zu stehen und haben sich mit der produktiven Arbeitskraft zu vereinen.

\section{Artikel 13}

Großes Singen und Blühen, große Wandzeitungen und große Debatten sind die neuen Formen der sozialistischen Revolution, die von den Volksmassen geschaffen wurden. Der Staat sorgt dafür, daß sich das Volk bei seinen Massenbewegungen dieser Mittel bedienen kann, um eine politische Lage zu schaffen, in der sowohl Demokratie als auch Zentralismus, sowohl Disziplin als auch Freiheit, sowohl vereinter Wille als auch individuelle Wohlfahrt und eine politische Situation der Lebensbegeisterung besteht, auf daß die Diktatur des Proletariats weiterhin konsolidiert wird.

\section{Artikel 14}

Der Staat gewährleistet das sozialistische System, unterdrückt alle verräterischen und konterrevolutionären Aktivitäten und bestraft alle Verräter und Konterrevolutionäre. Der Staat sorgt dafür, daß nach Maßgabe des Gesetzes Grundherren, reiche Bauern, reaktionäre Kapitalisten, Konterrevolutionäre und andere unerwünschte Elemente ihre politischen Rechte eine Zeitlang verlieren. Gleichzeitig eröffnet er ihnen einen Weg, ihren eigenen Lebensunterhalt zu verdienen, um ihnen so eine Möglichkeit zu geben, sich selbst durch Arbeit zu erziehen und Bürger zu werden, die ihren Lebensunterhalt durch ihre eigene Arbeit bestreiten können. 


\section{Artikel 15}

Volksbefreiungsarmee und Miliz sind Soldaten der Kinder von Arbeitern und Bauern unter der Führung der Kommunistischen Partei Chinas; sie sind die bewaffneten Kräfte der ganzen Nation. Die chinesische Volksbefreiungsarmee ist für immer eine Kampftruppe. Gleichzeitig ist sie aber auch eine Arbeits- und Produktionstruppe. Die Pflicht der bewaffneten Kräfte der Volksrepublik China besteht darin, die sozialistische Revolution und die Errungenschaften des sozialistischen Aufbaus zu verteidigen, die Souveränität, territoriale Integrität und Sicherheit des Staates zu schützen und die Subversion und Aggression des Imperialismus, des Sozio-Imperialismus und ihrer Lakaien abzuwehren.

\section{Abschnitt II: Die Staatsorgane}

\section{Erstes Kapitel: Der Nationale Volkskongreß}

\section{Artikel 16}

Der Nationale Volkskongreß ist das höchste Organ der Staatsgewalt unter der Führung der Kommunistischen Partei Chinas. Der Nationale Volkskongreß besteht aus Abgeordneten, die von den Provinzen, von den autonomen Regionen, von den zentral unterstellten Städten, von den Streitkräften und von den im Ausland lebenden Chinesen gewählt werden. Erforderlichenfalls kann eine Anzahl patriotisch gesinnter Persönlichkeiten zur Teilnahme eingeladen werden. Der Nationale Volkskongreß wird auf fünf Jahre bestellt, wobei die Zeit unter besonderen Umständen ausgedehnt werden kann. Der Nationale Volkskongreß tagt einmal im Jahr; unter besonderen Umständen kann die Sitzung aber vor dem bestimmten Datum abgehalten oder aber verschoben werden.

\section{Artikel 17}

Die Zuständigkeiten des Nationalen Volkskongresses sind folgende: Die Verfassung $\mathrm{zu}$ ändern, Gesetze zu beschließen, den Vorsitzenden des Staatsrates auf Empfehlung des Zentralkomitees der Kommunistischen Partei Chinas hin zu ernennen und abzuberufen, den Voranschlag sowie die Schlußabrechnung des Staatshaushaltes zu prüfen und $z u$ billigen und andere Funktionen zu übernehmen, die der Nationale Volkskongreß für notwendig hält.

\section{Artikel 18}

Der Ständige Ausschuß des Nationalen Volkskongresses ist das ständige Organ des Nationalen Volkskongresses. Seine Zuständigkeiten sind folgende: Er berät den Nationalen Volkskongreß, legt die Gesetze aus, beschließt Rechtsverordnungen, entsendet die Vertreter ins Ausland und ruft sie zurück, empfängt ausländische Gesandte, genehmigt Verträge mit dem Ausland und macht sie rückgängig. Der Ständige Ausschuß des Nationalen Volkskongresses besteht aus einem Vorsitzenden, dem Stellvertreter des Vorsitzenden und weiteren Mitgliedern, die vom Nationalen Volkskongreß gewählt und abberufen werden. 


\section{Zweites Kapitel: Der Staatsrat}

\section{Artikel 19}

Der Staatsrat ist die Zentrale Volksregierung. Er ist dem Nationalen Volkskongreß und seinem Ständigen Ausschuß verantwortlich und hat ihm über seine Tätigkeit Bericht abzulegen. Der Staatsrat besteht aus dem Ministerpräsidenten, seinem Stellvertreter, den Ministern und den Vorsitzenden der Kommissionen.

\section{Artikel 20}

Die Zuständigkeiten des Staatsrats sind folgende:

Er trifft auf der Grundlage der Verfassung, der Gesetze und der Erlasse Verwaltungsmaßnahmen, erläßt Verordnungen und Verfügungen. Er vereinheitlicht und führt die Arbeit der einzelnen Ministerien, der einzelnen Kommissionen und der lokalen Organe der Staatsverwaltung auf den verschiedenen Ebenen im ganzen Land; er formuliert den Nationalen Wirtschaftsplan sowie den Nationalen Haushalt und führt sie aus; er nimmt die Verwaltungsangelegenheiten des Staates wahr und übt andere Funktionen aus, die ihm vom Nationalen Volkskongreß oder vom Ständigen Ausschuß des Nationalen Volkskongresses übertragen werden.

\section{Drittes Kapitel: Die örtlichen Volkskongresse und die örtlichen Revolutions- komitees auf den verschiedenen Ebenen}

\section{Artikel 21}

Die örtlichen Volkskongresse auf den verschiedenen Ebenen sind die örtlichen Organe der Staatsmacht. Die Volkskongresse in den Provinzen und in den zentral unterstellten Städten haben eine Amtszeit von fünf Jahren; die Volkskongresse in Sonderdistrikten, Städten und Kreisen fungieren drei Jahre; die Volkskongresse in ländlichen Volkskommunen und Märkten amtieren zwei Jahre.

\section{Artikel 22}

Die örtlichen Revolutionskomitees auf den verschiedenen Ebenen sind die ständigen Organe der örtlichen Volkskongresse und gleichzeitig die Volksregierungen der jeweiligen Ebene. Ein örtliches Revolutionskomitee besteht aus dem Vorsitzenden, seinen Stellvertretern und weiteren Mitgliedern, die vom Volkskongreß auf der jeweiligen Ebene gewählt und abberufen werden. Ein örtliches Revolutionskomitee ist dem Volkskongreß der jeweiligen Ebene und dem Staatsorgan auf der nächsthöheren Ebene verantwortlich und hat ihnen über seine Arbeit zu berichten.

\section{Artikel 23}

Die örtlichen Volkskongresse auf den verschiedenen Ebenen und die örtlichen Revolutionskomitees, die von den örtlichen Volkskongressen gewählt werden, gewährleisten in ihrem Zuständigkeitsgebiet die Durchführung der Gesetze und Erlasse, entfachen nach einheitlichem staatlichen Plan die Aktivität auf den verschiedenen lokalen Ebenen, übernehmen die Führung über die örtliche sozialistische Revolution und den sozialistischen Aufbau, prüfen und genehmigen die örtlichen Haushalte, bewachen die revolutionäre Ordnung und schützen die Rechte der Bürger. 


\section{Viertes Kapitel: Die Organe der Selbstverwaltung in den Gebieten mit nationaler Autonomie}

\section{Artikel 24}

Die Organe der Selbstverwaltung in den autonomen Regionen, autonomen Bezirken und autonomen Kreisen, die alle Gebiete mit nationaler Autonomie darstellen, sind die Volkskongresse und die Revolutionskomitees. Die Organe der Selbstverwaltung in Gebieten mit nationaler Autonomie sind berechtigt, zusätzlich zu den Zuständigkeiten der örtlichen Staatsorgane, wie sie in Abschnitt II Kapitel 3 niedergelegt sind, auch noch Selbstverwaltung nach Maßgabe der Gesetze auszuüben. Die höheren Staatsorgane auf den verschiedenen Ebenen sind angehalten, die Ausübung der Selbstverwaltung durch Organe der Selbstverwaltung in den Gebieten mit nationaler Autonomie in vollem Umfang zu schützen und die verschiedenen Minderheiten bei ihren Bemühungen um sozialistische Revolution und um sozialistischen Aufbau zu unterstützen.

\section{Fünftes Kapitel: Organe der Rechtsprechung und der Staatsanwaltschaft}

\section{Artikel 25}

Die Aufgaben der Rechtsprechung werden von dem Obersten Volksgericht, von den örtlichen Volksgerichten und von den besonderen Volksgerichten ausgeübt. Die Volksgerichte auf den verschiedenen Ebenen sind den Volkskongressen und ihrem Ständigen Ausschuß auf der entsprechenden Ebene verantwortlich und haben ihnen über ihre Arbeit zu berichten. Die Ständigen Ausschüsse der Volkskongresse auf den verschiedenen Ebenen sind zuständig für die Ernennung und die Abberufung der Gerichtspräsidenten auf den entsprechenden Ebenen. Die Ausübung der Rechtsprechung und der strafverfolgenden Gewalt soll der Massenlinie folgen. Ernsthafte konterrevolutionäre und kriminelle Fälle sind in Massendiskussionen und Massenkritiken zu behandeln.

\section{Abschnitt III: Grundrechte und Grundpflichten der Bürger}

\section{Artikel 26}

Die fundamentalsten Rechte und Pflichten der Bürger sind folgende: Sie haben den Vorsitzenden Mao Tse-tung und seinen engen Waffenkameraden, den Stellvertretenden Vorsitzenden Lin Piao zu unterstützen; zu unterstützen haben sie ferner die Führung durch die Kommunistische Partei Chinas, die Diktatur des Proletariats und das sozialistische System. Ferner haben sie sich an die Verfassung und an die Gesetze der Volksrepublik China zu halten. Die Verteidigung des Vaterlandes und der Widerstand gegen Aggressionen gehören zu den heiligen Pflichten jedes Bürgers; es ist ehrenhafte Pflicht der Bürger, den Militärdienst nach Maßgabe des Gesetzes auszuüben.

\section{Artikel 27}

Bürger, die das 18. Lebensjahr erreicht haben, sind aktiv und passiv wahlberechtigt. Ausgenommen sind nur solche Personen, denen diese Rechte kraft Gesetzes abgesprochen wurden. Die Bürger haben das Recht auf Arbeit und das Recht auf Ausbildung. Arbeiter haben das Recht auf Ruhe und Freizeit und das Recht 
auf materielle Unterstützung im Alter und im Falle von Krankheit oder Arbeitsunfähigkeit. Männer und Frauen sind gleichberechtigt. Der Staat schützt die Ehe, die Familie sowie Mutter und Kind. Der Staat schützt die Rechte und Interessen der Chinesen im Ausland.

\section{Artikel 28}

Die Bürger haben Redefreiheit, Korrespondenzfreiheit, Publikationsfreiheit, Versammlungsfreiheit, Vereinigungsfreiheit, Demonstrationsfreiheit und das Recht zum Streiken. Sie haben ferner das Recht, einer Religion anzugehören sowie das Recht, keiner Religion anzuhängen. Auch ist es ihnen freigestellt, den Atheismus zu verkündigen. Die Freiheit der Person und die der Wohnung sind unverletzlich. Kein Bürger darf ohne Gerichtsbeschluß oder ohne Genehmigung der Sicherheitsorgane festgenommen werden.

\section{Artikel 29}

Die Volksrepublik China gewährt Asyl jedem Ausländer, der wegen Unterstützung einer gerechten Sache, wegen Teilnahme an revolutionären Aktivitäten oder wegen seiner wissenschaftlichen Tätigkeit verfolgt wird.

\section{Abschnitt IV: Nationalflagge, Nationalwappen, Hauptstadt}

\section{Artikel 30}

Die Staatsflagge der Volksrepublik China besteht aus einer roten Fahne mit fünf Sternen. Das Staatswappen der Volksrepublik China zeigt in der Mitte das von fünf Sternen überstrahlte Tor des Himmlischen Friedens und ist von Ähren und einem Zahnrad umrahmt. Die Hauptstadt der Volksrepublik China ist Peking. 